\title{
Comparison of two beam angular optimization algorithms guided by automated multicriterial IMRT
}

\author{
T. Ventura ${ }^{1,2,3}$, H. Rocha ${ }^{3,4}$, B. C. Ferreira' ${ }^{3,5}$, J. Dias ${ }^{3,4}$ and M. C. Lopes ${ }^{1,2,3}$ \\ ${ }^{1}$ Physics Department of University of Aveiro, Campus Universitário de Santiago, 3810-193 Aveiro, \\ Portugal \\ ${ }^{2}$ Medical Physics Department of Instituto Português de Oncologia de Coimbra Francisco Gentil, \\ EPE, Avenida Bissaya Barreto, no 98, 3000-075 Coimbra, Portugal \\ ${ }^{3}$ Institute for Systems Engineering and Computers at Coimbra, Rua Sílvio Lima, 3030-290 \\ Coimbra, Portugal \\ ${ }^{4}$ Economy Faculty of University of Coimbra and Centre for Business and Economics Research, \\ Avenida Dias da Silva 165, 3004-512 Coimbra, Portugal \\ ${ }^{5}$ School Health Polytechnic of Porto, Rua Dr. António Bernardino de Almeida, 400, 4200-072 \\ Porto, Portugal
}

\begin{abstract}
Purpose

To compare two beam angle optimization (BAO) algorithms for coplanar and non-coplanar geometries in a multicriterial optimization framework.

\section{Methods}

40 nasopharynx patients were selected for this retrospective planning study. IMRT optimized plans were produced by Erasmus-iCycle multicriterial optimization platform. Two different algorithms were used to address BAO: the combinatorial iterative algorithm embedded in Erasmus-iCycle, algorithm $i$, and an algorithm based on a pattern search method, algorithm $B$. Plans quality evaluation and comparison were performed with SPIDERplan. Statistically significant differences were assessed with a randomized block design ANOVA test and a post-hoc multiple comparison test using the Tukey method. Single pair comparisons were evaluated with t-test.
\end{abstract}

\section{Results}

Globally, both algorithms presented near equivalent quality scores, with algorithm $B$ presenting a marginally better performance than algorithm $i$. Although there are not significant differences when average results are considered, a case-by-case analysis can reveal important differences for some patients. For plans using only coplanar gantry angles, the optimized beam distribution with algorithm $i$ is more asymmetric than with algorithm $B$ beams where beams are more evenly distributed. For non-coplanar beam optimization, larger deviations from coplanarity were obtained with algorithm $i$ than with algorithm $B$. Plan quality increased with the number of beams and with algorithm $B$ noncoplanar geometries have also shown to be advantageous.

\section{Conclusion}

Almost all plans presented a high quality, benefiting from multicriterial and beam angular optimization. On average algorithm $B$ showed slightly better results than algorithm $i$. However, BAO using algorithm $B$ can be important for particular patients' cases.

Keywords: beam angular optimization, multicriteria optimization and plan assessment 


\section{Introduction}

Intensity-modulated radiation therapy (IMRT) is becoming the standard technique in radiation therapy. Non-uniform intensity fields from multiple directions are used to generate high conformal dose distributions to the tumour. The plan objectives are usually described by physical or biological descriptors that are typically incorporated in an objective function that will guide the Fluence map optimization (FMO) procedure by scoring the goodness of the plan [1]. Searching methods such as linear least squares [2], gradient descent [3] or simulated annealing [4] are used to compute the intensity pattern that provides the best possible trade-off between conflicting planning goals. A trial-and-error iterative manual tuning of plan parameters (like weights, objectives or beam angles) may be necessary to achieve an acceptable plan. One important difficulty in this iterative process is the fact that it is not possible to know the impact that changing one given parameter will have in the treatment plan, or what are the interdependencies that exist between the different parameters. This iterative process is thus mainly guided by the empirical knowledge of the planner. Furthermore, it is also not possible to link the parameters' values with the desired clinical planning goals. As a result, it is not possible to guarantee that the trial-and-error optimization process will lead to an optimal plan. This process is also more or less time-consuming depending on the case complexity and mostly on the planner skills [5].

Multi-criteria optimization (MCO) methods come up as a natural option to support IMRT treatment planning decision making process. Instead of a single objective function, multiple objective functions, resulting from the goals assigned to targets and normal tissues, are simultaneously maximized (or minimized). As most of the times it is not possible to find a single feasible solution that is simultaneously the best one for every objective function [6,7], a set of optimal plan solutions containing the best possible trade-offs between objectives are presented to the decision maker.

Beam angle selection plays also an important role in IMRT optimization. An appropriate beam angle assembly choice, based on a mathematical criterion rather than on the planner experience or on equidistant coplanar arrangement solutions, may lead to important enhancements in the final plan dose distribution solution [8]. Plan quality improvements can be even more significant if non-coplanar directions are included in the optimization process [9]. Mathematically, the beam angular optimization (BAO) problem can be described as a highly non-convex multi-modal optimization problem with many local minima [10-12], which ideally requires methods with few computing iterations and able to avoid getting trapped in local minimum. BAO solutions are often non-intuitive, so it is important to use optimization approaches that are reliable considering their capacity of delivering optimal solutions [13[13]].

The BAO problem can be addressed in two different ways. One possibility is to decouple the beam angle selection from the FMO. In this case, BAO is driven by geometrical measures (e.g., beam's-eye view metrics) or by methods that require prior knowledge of the problem $[8,14]$. Although computationally efficient, these methods do not fully account for the interplay of beam angles and beamlets weights. Another possibility is to jointly address BAO and FMO problems. FMO optimal solutions are used to assess the beams set plan quality during the BAO [12]. An exhaustively combinatorial search for the best ensemble of beams over a discretized space search defined with all possible beam incidences can be done using heuristic methods [11,12,15-19]. However, as this formulation is considered a nondeterministic polynomial time hard problem [20], alternative combinatorial approaches have also been developed. The iterative BAO methods wherein the beams are iteratively subtracted [21] or added $[22,23]$ to a beam ensemble, decreasing significantly the possible number of combinations, are one the most well-known examples. Also BAO methods based on the continuous exploration of the solutions search space have been explored as an alternative to the combinatorial BAO, namely using pattern search methods $[9,24]$, or considering a parallel multistart derivative-free optimization framework $[25,26]$.

In the present work, the BAO problem is addressed using two algorithms, one belonging to the discrete combinatorial [23] type and the other to the continuous space search approach 
optimization [24] class. Both algorithms use the FMO objective function to guide the BAO process. They were compared over a set of 40 nasopharyngeal cancer (NPC) clinical cases. The correspondent IMRT plans were optimized by an automated MCO calculation engine developed by Breedveld et al. [27]. Coplanar and non-coplanar geometry scenarios and different number of beams incidences in treatment delivery were considered in this retrospective planning study. The plans were assessed and compared using SPIDERplan [28], that evaluates the quality of the dose distribution through an intuitive graphic representation and an associated score function that are based on dose prescription aims.

\section{Materials and methods}

\subsection{Patient data}

Forty NPC cases treated with IMRT were selected for this study. All selected cases had simultaneous integrated boost prescription delivered in 33 fractions, 70.0 Gy to the tumour planning target volume (PTV) and a dose ranging between 54.0 Gy and 59.4 Gy according to the associated risk disease level to the lymph nodes PTV. Some patients had one or more adenopathies that were also prescribed with 70Gy. Spinal cord, brainstem, retinas, lens, optical nerves, chiasm, pituitary gland, ears, parotids, oral cavity, temporomandibular joints, mandible, oesophagus, larynx, brain, thyroid and lungs were also contoured by the radiation oncologist. The organs-at-risk (OAR) tolerance doses were established in agreement with institutional protocols for the nasopharyngeal pathology.

\subsection{Plan generation and optimization}

FMO for all plans was achieved by using Erasmus-iCycle IMRT multicriterial optimization engine [27]. Guided by a wish-list, containing clinical constraints and prioritized objectives, a constraint-based method, $2 p \varepsilon c$ method, is used to automatically generate a single Pareto optimal IMRT solution for a given set of beams. The wish-list template, built for NPC cases, contains clinical constraints and prioritized objectives that were divided in two optimization levels, according to the clinical tolerance doses, the proximity between PTVs and OARs and its impact on the dose distribution. This configuration with a progressive dose optimization structure is ideal for complex sites, like the NPC cases. It intends to avoid possible limitations that may arise when a dose value achieved in an OAR with a high priority restricts the optimization of another one with a lower priority. The objectives associated with the PTVs were assigned with the Logarithmic Tumour Control Probability (LTCP) function, which is regulated by a cell sensitivity parameter ( $\alpha$ ). For this study, an a value of 0.75 was applied to guarantee that at least $98 \%$ of the PTV volume receives $95 \%$ of the prescription dose $\left(D_{p}\right)$. To allow the minimization of lower prioritized objectives, a LTCP sufficient value of 0.5 was defined. The remaining objectives were defined according to the OAR type. For organs with a serial architecture a maximum dose objective was defined. For parallel architectures a mean dose objective was applied. Also, the dose of non-vital OARs, such as lens, optics, retinas, brain or pituitary gland, was minimized using with the generalized Equivalent Uniform Dose (gEUD) function with a value of the tissue-specific parameter that describes the volume effect (a) equal to 12.

\subsection{Beam angular optimization}

For BAO of coplanar and non-coplanar beam geometries two different methods were tested. In the first method, developed by Breedveld et al. [29] and implemented within Erasmus-iCycle, BAO is integrated in the plan optimization framework, considering a discretization of the search space. Plan generation is done by iteratively adding beams with an optimal orientation into the plan. For a given beam arrangement, all possible candidates will be combined with the beams already selected for the plan and the candidate beam that achieves the lowest score of the fluence optimization problem is added. New beams will be sequentially added until no significant dose distribution 
improvements are achieved or if the maximum number of beams initially defined is reached.

The second approach, developed by Rocha et al. [24], explores the continuous BAO search space using a pattern search method. These class of methods are directional direct search methods and thus do not require the use of derivatives to minimize the objective function. To assure a more effective search for the best objective function local minimum, a set of points spanning as much as possible the entire search space is defined in a preliminary step of the pattern search optimization. Thus, the objective function values of a set of plans with equally spaced orientations that span the entire beam angle search space are determined. The pattern search optimization is organized around two steps: the search step and the poll step. It starts with the search step where any (global) strategy can be used to improve the best objective function value. In this implementation, minimum Frobenius norm quadratic models were used to perform a search over the whole search space [30]. These quadratic models are based on the beam angle sets already considered. If the corresponding objective value is lower than the best objective function minimum value, the search step was successful and is repeated. Otherwise the optimization method proceeds to the poll step, where the current best solution is locally improved using the concept of positive basis. If this step fails to obtain a decrease in the objective function value, the step-size parameter is reduced. If the step-size becomes smaller that the defined limit the process stops, otherwise a new loop of the algorithm is performed starting a new search step. When the maximum number of iterations is reached, the pattern search optimization will stop.

\subsection{Study design}

IMRT plans were automatically generated in iCycle for all NPC cases. Based on the defined wish-list, plan optimization was performed using 7 coplanar equidistant beams ( $d 7)$ corresponding to the standard clinical option. In a second phase, plan optimization was done by applying beam angular optimization. Breedveld et al. [29] (algorithm I) and Rocha et al. [24] (algorithm B) beam angular optimization algorithms were used to generate IMRT plans with 5,7 and 9 beams ( $i 5, i 7, i 9$ and $B 5$, $B 7, B 9$, respectively). For both algorithms, coplanar and non-coplanar beam geometries were considered.

\subsection{Plan assessment and comparison}

Plan assessment and comparison were performed using an independent graphical method developed by Ventura et al. [28]. SPIDERplan, is based on a scoring approach that considers both target coverage and individual OAR sparing. In SPIDERplan framework, targets and OARs are divided into groups depending on their clinical priorities. A score is determined for each structure based on pre-defined planning objectives and relative weights. Global plan score is determined as a weighted sum of the structures' individual scores over all groups. All dosimetric plan information is graphically represented in customized radar plots. Evaluation of plan quality can be done globally displaying all structures (Structures Plan Diagram - SPD) and groups of structures (Group Plan Diagram - GPD). A more detailed group evaluation can also be done with the partial group plots (Structures Group Diagrams - SGD), where only the structures of that group are represented. As for the SPD and GPD, a partial group score complementing the graphical assessment is determined for each SGD.

For this study, all delineated structures were grouped according to their location and clinical importance into: PTV group (PTVs), Critical group (spinal cord and brainstem), Optics group (chiasm, optical nerves, retinas and lens), DigestOral group (parotids, oral cavity, oesophagus and larynx), Bone group (temporal mandibular joint, mandible and ear canals) and Other group (brain, pituitary gland, thyroid and lungs). To each group a relative weight of $50 \%, 30 \%, 10 \%, 5 \%, 3.5 \%$ and $1.5 \%$, respectively, was pre-assigned by the radiation oncologist. Within each group, the same weight was attributed each structure of that group. The score of each structure is determined considering the ratio between the clinical tolerance criteria and the planned dose. Thus, a value of one is expected 
if the dose for that structure is equal to the respective tolerance value. When a better organ sparing or target coverage is obtained, a score less than one will be obtained. Optimal scores will converge to the centre of the radar plot [28].

\subsection{Statistical Analysis}

Statistical comparisons of the mean scores associated with each BAO algorithm and geometry sets were performed using IBM SPSS software, version 25. As the same set of patients is used to perform IMRT optimization applying the two BAO algorithms and different geometric settings, it was assumed that the samples were dependent. As the number of patients selected for this retrospective study is greater than 30 , it was also considered that the samples follow a normal distribution. Statistically significant differences between the families of test were assessed with a randomized block design ANOVA test and, if applicable, a post-hoc multiple comparison test using the Tukey method. Single pair comparisons were statistically evaluated with the t-test. A level of significance of $5 \%$ was considered for all statistical tests.

\section{Results}

\subsection{Beam angle distribution}

The frequency analysis of the beam angle distribution of algorithms $i$ and $B$ for 5,7 and 9 beams is shown in Figure 1 (coplanar setting) and Figure 2, (non-coplanar setting).

For the coplanar $\mathrm{BAO}$, circular diagrams composed of three concentric rings with an angle section resolution of $10^{\circ}$ were used to represent the relative frequencies of the beam angle distribution obtained for each of the two algorithms, Figure 1. The inner ring of the circular diagrams shows the beam angle distribution of the BAO with 5 beams, the middle ring corresponds to the optimization with 7 beams and in the external ring the 9 beams results are shown. Each ring was divided into eight regions commonly applied in the clinical routine for gantry angles: the anterior region defined between $340^{\circ}$ to $20^{\circ}$, the left oblique anterior (LOA) region defined between $20^{\circ}$ to $70^{\circ}$, the left lateral $(\mathrm{LL})$ region defined between $70^{\circ}$ to $110^{\circ}$, the left oblique posterior (LOP) region defined between $110^{\circ}$ to $160^{\circ}$, the posterior region defined between $160^{\circ}$ to $200^{\circ}$, the right oblique posterior $(R O P)$ region defined between $200^{\circ}$ to $250^{\circ}$, the right lateral $(R L)$ region defined between $250^{\circ}$ to $290^{\circ}$ and the right oblique anterior $(R O A)$ region defined between $290^{\circ}$ to $340^{\circ}$. The beam

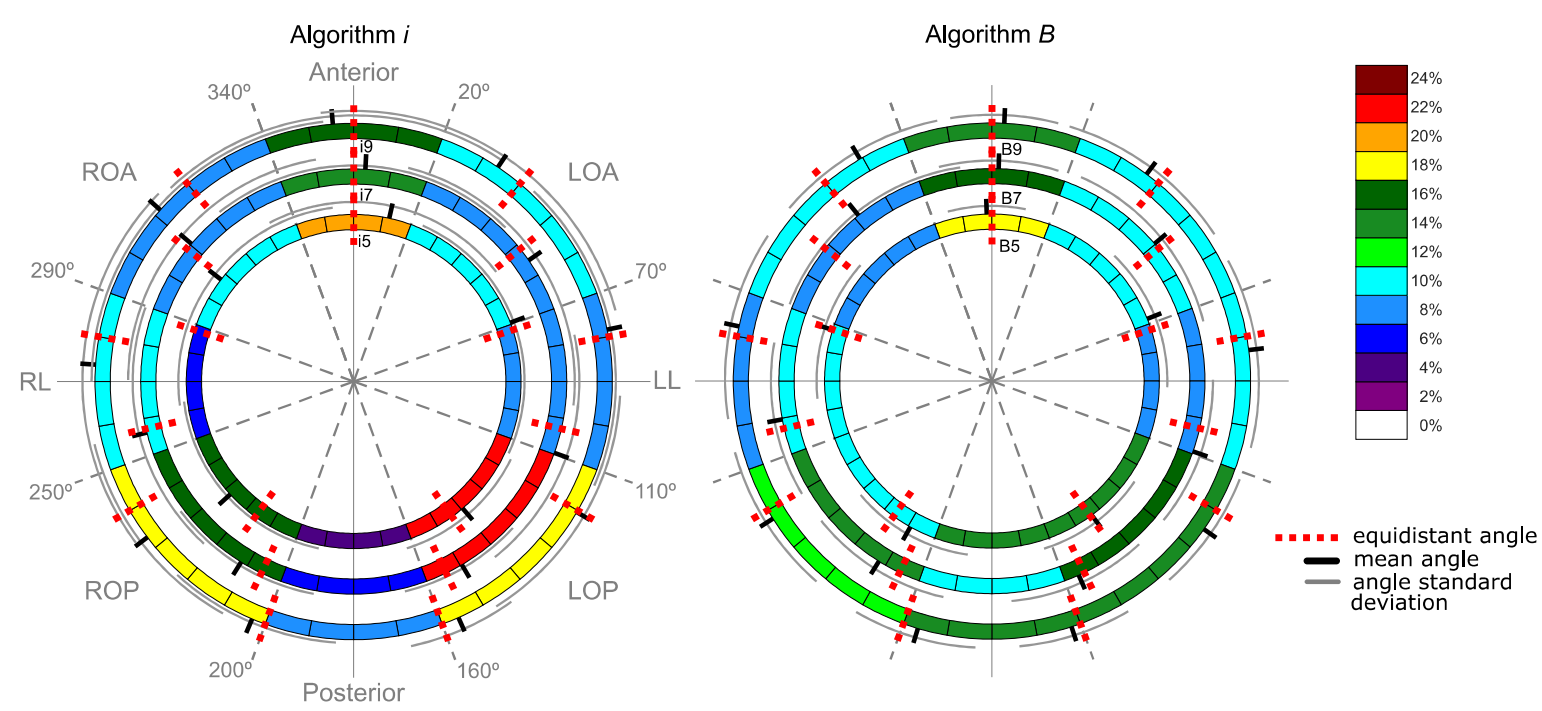

Figure 1 - Angular representation of the relative frequencies of the coplanar BAO of algorithms $i$ and $B$ for 5 , 7 and 9 beams. The colour represents the relative frequencies obtained for each angle section: a hot colour is associated to a high relative frequency and a cold colour to a low relative frequency value. 
angles for the equidistant beam angle solution (red dash line) and the mean angle incidences (black solid pointers) with the associated standard deviation (grey solid arcs) are also represented in each circular beam diagram.

The two coplanar BAO approaches presented distinct beam angular distribution patterns. In algorithm $i$ (i5, i7 and $i 9$ ), based on an iterative BAO framework, the beam angular distribution across the regions was asymmetric with preferred regions very well defined as the LOP, the anterior or the ROP regions. The relative frequency values were comprehended between $4 \%$ and $22 \%$. The higher

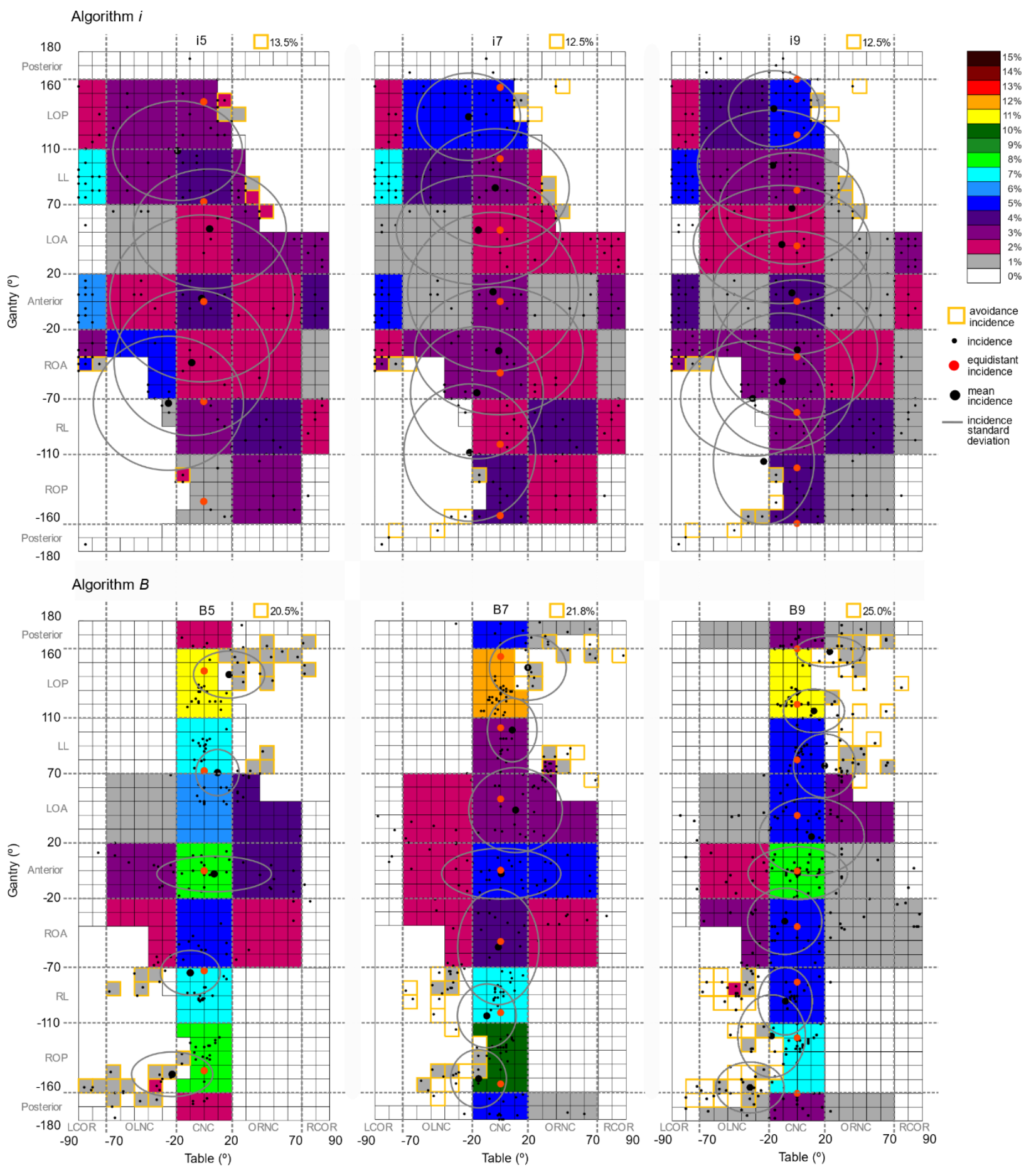

Figure 2 - Representation of the relative frequencies of the non-coplanar BAO of algorithm $i$ and $B$ for 5,7 and 9 beams 
relative frequency values, corresponding to the preferred irradiation directions, were the LOP region and the anterior region in i5 set and also the ROP region in $i 7$ and i9 sets. For algorithm $B(B 5, B 7$ and $B 9$ ), a more evenly angular distribution was obtained, with relative frequency values ranging between $8 \%$ and $18 \%$. For all sets, the anterior, the posterior and the LOP regions presented the higher relative frequency values. For both algorithms, anterior-oblique and lateral orientations were not often selected as good irradiation directions regardless the number of beams used. The difference between the optimal mean angle and the correspondent equidistant beam was $0.8^{\circ} \pm 34^{\circ}$ for algorithm $i$ and $1.5^{\circ} \pm 19.9^{\circ}$ for algorithm $B$.

In Figure 2, for the non-coplanar BAO modality, the gantry angle distribution and the table angle distributions are presented by a relative frequency 2D-map with a squared grid resolution of $10^{\circ}$. The gantry angles axis (vertical) was divided in the same groups defined as for the coplanar case. The table angles axis (horizontal) was grouped into five regions: the left coronal (LCOR) region defined the rotation of the table between $-90^{\circ}$ and $-70^{\circ}$, the oblique left non-coplanar (OLNC) region defined between $-70^{\circ}$ and $-20^{\circ}$, the central non-coplanar (CNC) region defined between $-20^{\circ}$ and $20^{\circ}$, the oblique right non-coplanar (ORNC) region defined between $20^{\circ}$ and $70^{\circ}$ and the right coronal (RCOR) region defined between $70^{\circ}$ and $90^{\circ}$. For simplification each region, composed of a set of gantry and table angles, will first be named with the gantry region followed by the table region (for instance, posterior_CNC). In Figure 2, the beams position for the equidistant coplanar beam plans are shown by the red dots, the individual beam incidences obtained by angular incidences by small black dots, the correspondent mean angle incidences by the large black dots and the associated standard deviation by the grey ellipses. The avoidance incidences, corresponding to potential collisions between gantry and couch were represented by the yellow grid squares.

The two non-coplanar BAO algorithms presented again distinct beam distribution patterns. In algorithm $i$, most of the beams were uniformly distributed over the space, with relative frequencies ranging between $0 \%$ (white squares in Figure 2) and 7\% (cyan). Interestingly, the preferred irradiation direction selected by algorithm $i$ where almost neglected by algorithm $B$ where relative frequency values of less than $1 \%$ were obtained. In algorithm $B$, with relative frequency values ranging between $0 \%$ and $13 \%$, it is possible to define a pattern for the beam's distribution. In fact, the non-coplanarity is almost confined to table angulation between $-20^{\circ}$ to $20^{\circ}$, corresponding to the $\mathrm{CNC}$ region. The remaining regions present relative frequency values inferior to $2 \%$.

The average beam incidences, and especially the standard deviation values, for both algorithms are quite different. Graphically, this can be perceived in Figure 2 by the clear separation between the ellipses for algorithm $B$ while for algorithm $i$ the standard deviations ellipses overlap each other. Furthermore, the distance between the mean incident angles (large black dots) and the correspondent equidistant solution (red dots) are closer from algorithm $B$ than from algorithm $i$.

\subsection{SPIDERplan scores analysis}

\subsubsection{Global plan analysis}

The values of the global plan score, implemented in SPIDERplan, for the d7 plans (equidistant beams) and coplanar and non-coplanar BAO of algorithms $B$ and $i$ for 5,7 and 9 beams are shown in Figure 3a. The mean SPIDERplan global plan scores ranged between 0.901 and 0.947 . The lowest mean plan scores, i.e. the plans with better overall score performance, were obtained by $B 9$ non-coplanar (B9nC) and B9 coplanar (B9C) sets. i9c plans attained the third better score, while i9nc set only achieved the eighth better score immediately below all plans using 7 beams. $B 5 c$ and $B 5 n c$ plans, respectively, obtained a better performance than $i 7 n c$. The highest mean global plan scores, and therefore the worst overall plan performances, were obtained with the $i 5 n c$ and $i 5 c$ sets.

The statistical analysis that was made allowed the identification of pairs of algorithms and beam angle configurations such that the generated treatment plans cannot be considered as being different from a statistical significance point of view. The results of the statistical analysis and the resulting $p$-value of each comparison led to seven subsets, grouping the algorithms that did not 

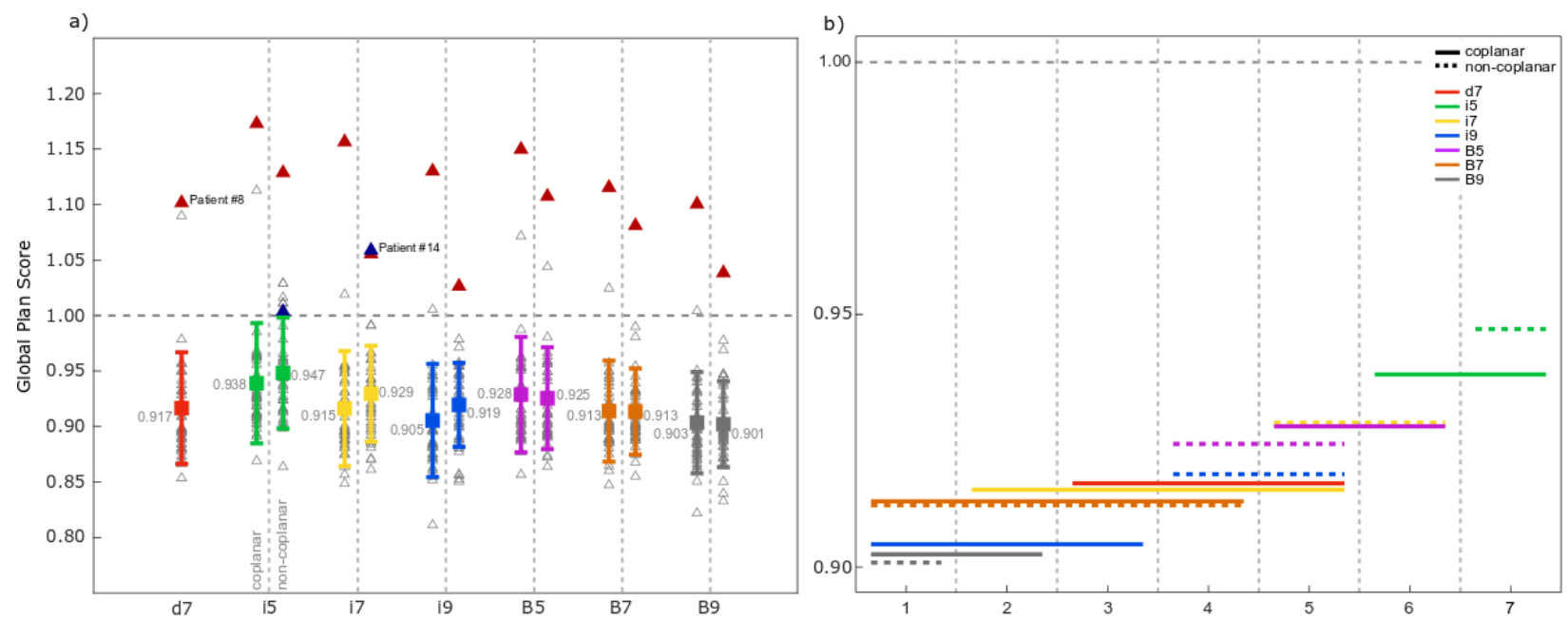

Figure 3 - a) SPIDERplan Global Plan scores, corresponding to all 40 clinical cases (triangles), for d7 and for coplanar and non-coplanar BAO of algorithms $i$ (coplanar plans: i5c, i7c, i9c, non-coplanar plans: i5nc, i7nc and i9nc) and $B(B 5 c, B 7 c, B 9 c, B 5 n c, B 7 n c$ and $B 9 n c)$. b) Homogenous subsets resulting from post-hoc multiple comparisons test using the Tukey method with a level of significance of $5 \%$ of the SPIDERplan global plan scores of each algorithm for coplanar and non-coplanar BAO.

present statistical significant differences. It was thus possible to build sets, as presented in Figure $3 b)$, such that each set will include similar treatment planning results. As an example, subset 1 shown in Figure 3b, with the lowest global plan scores, includes B9nc, B9c, i9c, B7nc, B7c, and i7c meaning that the quality of these plans is statistically equivalent. Statistically significant differences were found between plans B9nc (positioned in subset 1) and plans i9nc (belonging to subsets 4 and 5); and between $B 9 c$ and $d 7$ and $i 9 n c$ plans. These results show that plans generated by BAO do not significantly differ from $\mathrm{d} 7$ plans using equidistant beams (indicated by all plans overlapping the red solid line representing $d 7$ plans). However, plan quality improved when the number of beams increased to 9 beams: as in $i 9 c$ or B9c and B9nc (subset 1) compared to 7 beams: i7nc or $d 7$. Also, while a statistically significant difference in plan quality was found between non-coplanar and coplanar plans using 9 beams whose positions were determined by algorithm i, for algorithm B, noncoplanarity brought no improvement in terms of plan quality. It is interesting to observe that, for algorithm $i$ and 9 beams, the 9 beam coplanar plans were better than the non-coplanar ones.

The overall superior performance of algorithm $B$ over algorithm $i$ was statistically significant $(p=0.000)$, Figure $4 \mathrm{f}$. The mean scores of coplanar and non-coplanar sets of algorithms $i$ and $B$ are compared in Figure $4 \mathrm{a}$ and Figure $4 \mathrm{~d}$, respectively. For algorithm $i$, the coplanar set had a lower mean score than the non-coplanar set $(\mathrm{p}=0.002)$ whereas for algorithm $B$, non-coplanar plans were statistically equivalent to coplanar ones $(p=0.960)$. Statistical significant differences were also found between non-coplanar plans optimized by algorithm $i$ and $B(\mathrm{p}=0.000)$, in favour of non-coplanar $B$ plans, (Figure $4 \mathrm{e}$ ). Figure $4 \mathrm{f}$ demonstrates that, globally, coplanar $B$ plans performed better than noncoplanar $i$ plans $(p=0.000)$.

\subsubsection{Group plan analysis}

The quality of the plans based on the BAO of algorithms $B$ and $i$ was assessed also using the information generated by SPIDERplan diagrams. Generally, the group score was in agreement with the analysis performed for the global plan score section. Almost all structure groups included in the optimization got mean scores below 1 , meaning that the clinical criteria were on average accomplished. The exception was the DigestOral group, where for the parotids and for the oral cavity planned doses surpassed tolerance doses. Differences in the mean group scores between noncoplanar plans of algorithm $i$ and the remaining plans and between 9 beams and 5 beams plans of 

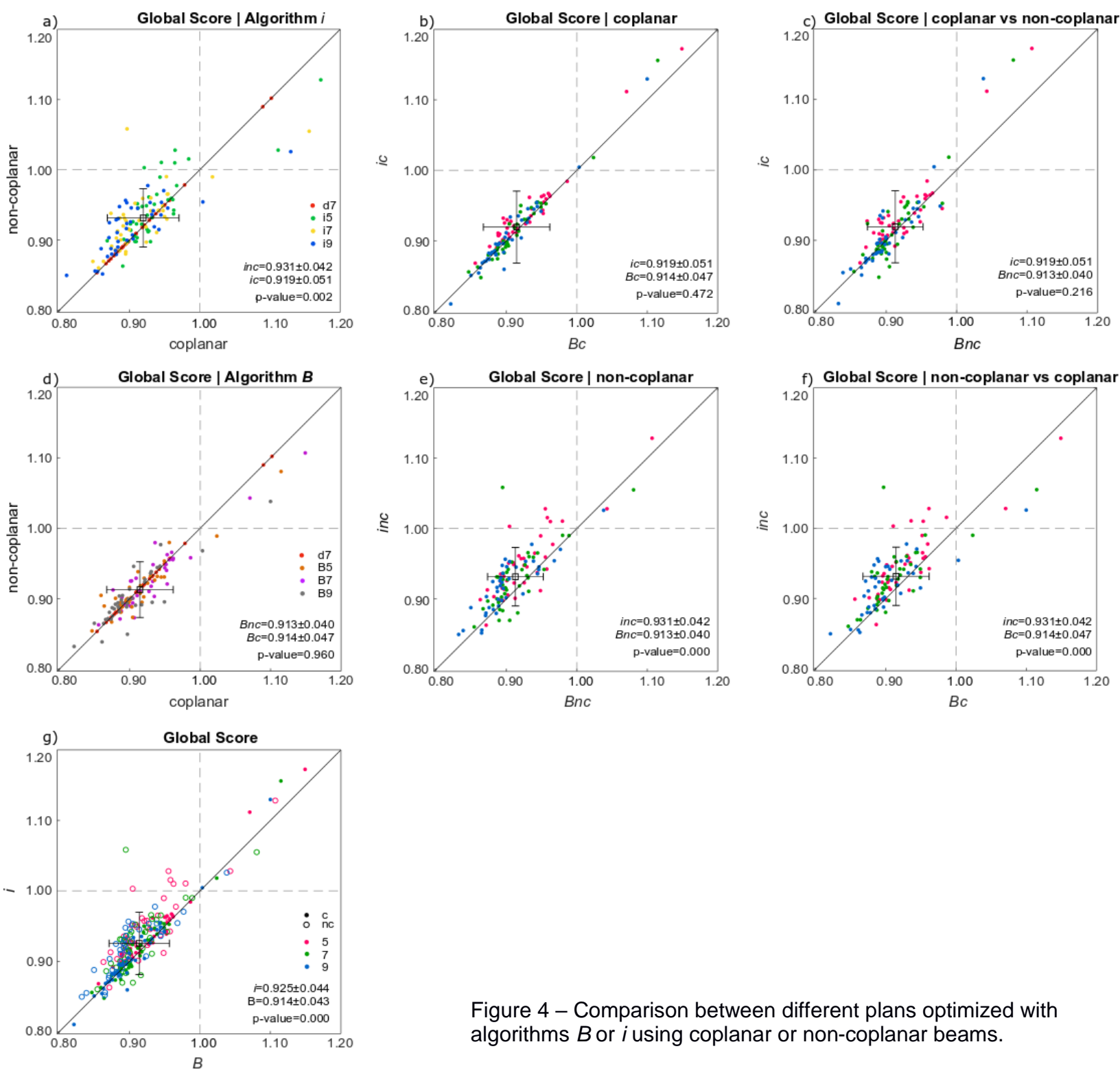

Figure 4 - Comparison between different plans optimized with algorithms $B$ or $i$ using coplanar or non-coplanar beams.

algorithms $i$ and $B$ were also obtained for the Optics and the DigestOral groups, respectively.

The plans with higher number of beams and a non-coplanar geometry tend to lead to dose distributions with better quality, i.e. higher PTV coverage and higher OAR sparing. Some exceptions were found for the Optics and Bone groups. In the Optics group, the best scores were found for the coplanar beam geometries of algorithm $B$ and the worst for the non-coplanar sets of algorithm $i$. For the Bone group, either the non-coplanar or the coplanar plans of algorithm $B$ achieved the best performances, while the coplanar and the non-coplanar sets of algorithm $i$ got the worst scores. Globally, algorithm $i$ presented better scores for the two most important groups (PTV and Critical group), while algorithm $B$ got the best scores for the remaining groups. However, the differences in plan quality for each structure group between the two algorithms were statistically significant just for the Optics and Bone groups, which included the OARs with the lowest clinical weight.

\subsubsection{Individual patient analysis}

The decision of which beam set-up should be used in a given patient must be well pondered and clinically assessed case by case. In Figure 3a, two patients (patient \#8 and \#14) were identified with notorious high scores (worst plan quality). For patient \#8 (red triangles in Figure 3a), all plans 
obtained a global score superior to 1 and presented mean percent differences between the coplanar and the non-coplanar sets for algorithms $i$ and $B$ of $-8 \%$ and $-5 \%$, respectively. For patient \#14 (blue triangles in Figure 3a), two plans exceeding the score threshold defined for SPIDERplan, presented an apparent contradictory score difference, wherein plan i5nc was better (lower score) than plan i7nc. The assessment of plan quality for patients \#8 and \#14 is presented in Figure 5 and Figure 6 , using the GPD and the SGDs of SPIDERplan. Plans using equidistant beam angle (d7) and the plans with the best and worst global plan scores were selected for this individual analysis. One or more additional sets were also considered to emphasize some results of interest observed in each patient.

For patient \#8, the best global plan score was achieved by plan i9nc (global plan score of 1.026 ) and the worst global plan score, of 1.172 , was obtained with plan $i 5 c$, representing a $15 \%$

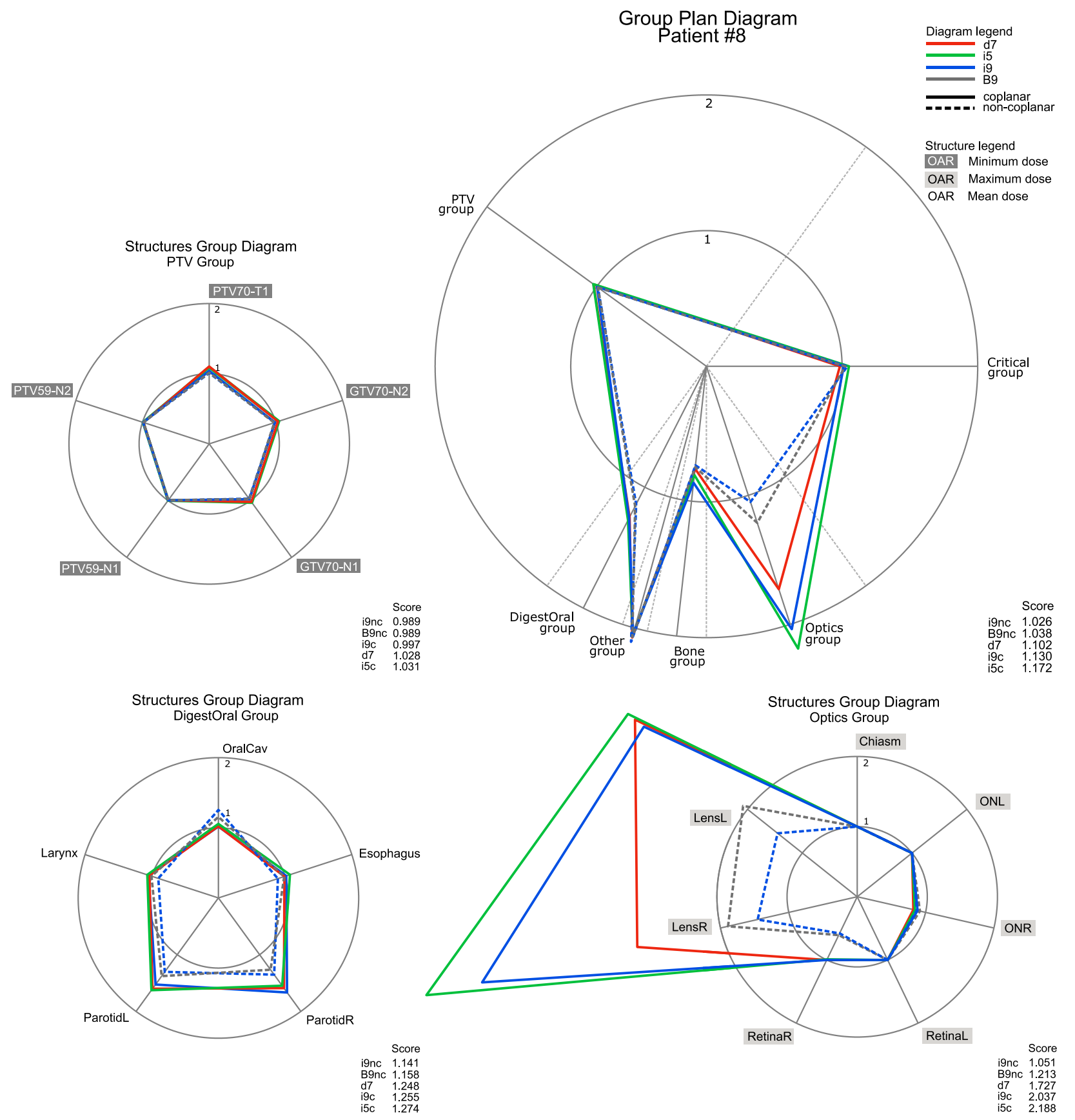

Figure 5 - SPIDERplan of patient number 8 and structures group diagram for PTV group, Optics group and DigestOral group 

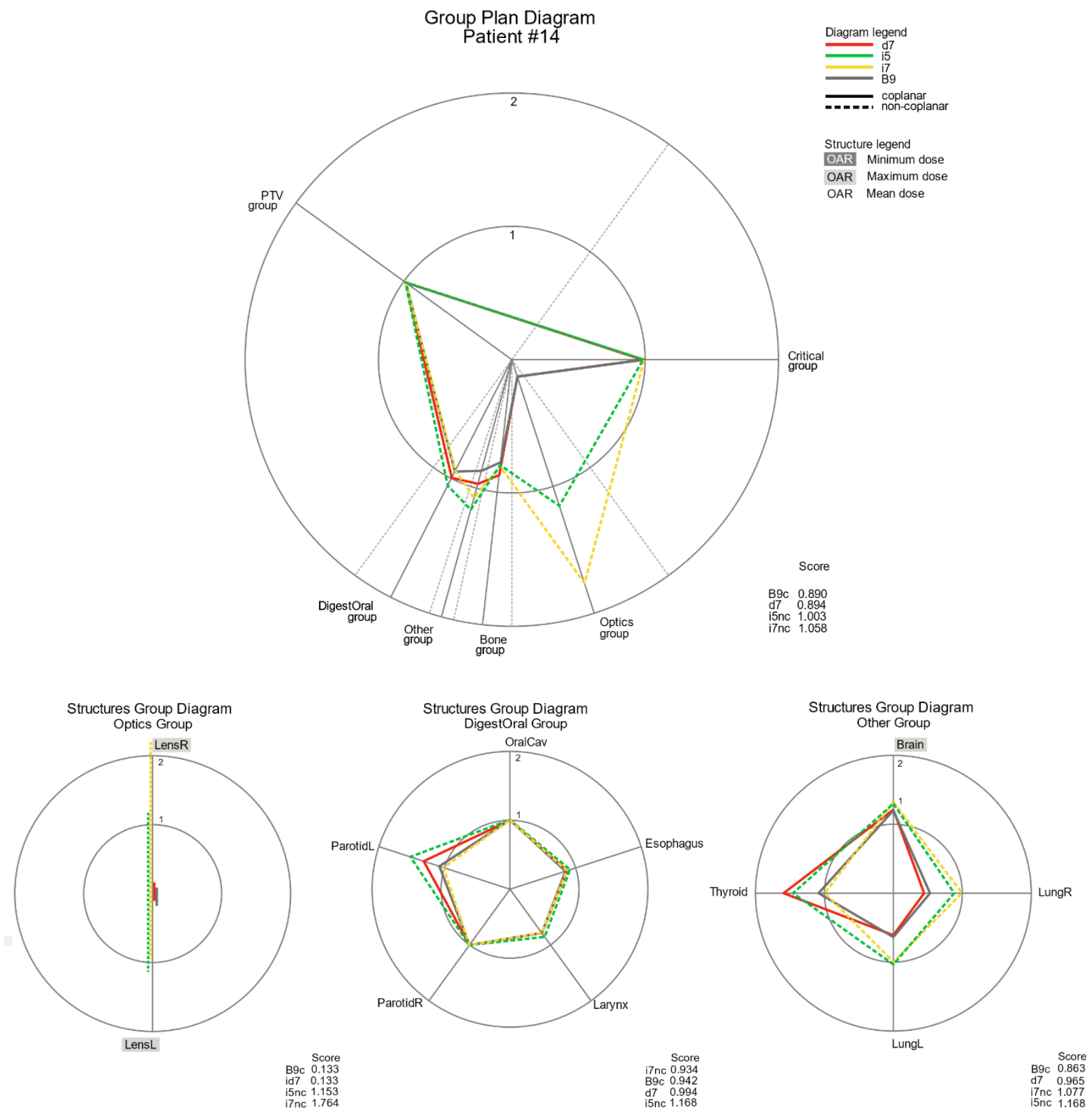

Figure 6 - SPIDERplan of patient number 14 and structures group diagram for Optics group, DigestOral group and Other group.

percent difference between the quality of these two plans. A percent difference of $-11 \%$ was obtained between the global plan scores of i9nc and i9c. These differences highlight the potential benefits that can arise from angular optimization including non-coplanar beam angle incidences. The largest difference between the tolerance and the planned dose was obtained for the Optics group and the DigestOral group (Figure 5). For the PTV group and in the Critical group some score values slightly higher than 1 were also obtained for some plans due to the proximity between the primary tumour mass, prescribed to $70 \mathrm{~Gy}$, and the retinas, the optical nerves, the chiasm, the brainstem, the ears and the oral cavity. The increase in the number of beams with non-coplanar geometries led to important improvements in the OAR sparing, especially in the lens and the parotids but also in PTV coverage. Nevertheless, these improvements were not extensible to all structures where even worst results were obtained for the oral cavity when 9 non-coplanar beams were used. 
For patient \#14, B9c presented the best global plan score and $i 7 n c$ the worst performance (Figure 6). A mean percent difference in the global score of $-10 \%$ was achieved when coplanar and non-coplanar sets of algorithm $i$ were compared. For algorithm $\mathrm{B}$, this mean percent difference was close to $0 \%$, meaning that for this patient the non-coplanarity did not bring any advantage for algorithm B. Significant differences between the considered plans can be identified for the lens (Optics group), the left ear (Bone group) and for the left parotid (Digest Oral group). All structures but the right lens presented better scores for a higher number of beams and/or non-coplanar geometry. For the right lens, however, i5nc presented a better score than $i 7 n c$. This configures a situation where a larger number of beams did not bring improvements to the overall plan quality. Analysing the specific anatomy of patient \#14, it is possible to observe that the primary PTV was well below the optical structures (chiasm, optical nerves, retinas and lens). This influenced the noncoplanar BAO process and probably the SPIDERplan analysis, since some of the considered clinical criteria could probably have been relaxed.

\section{Discussion}

In this work two BAO algorithms, $i$ and $B$, were evaluated and compared for NPC tumour cases. Forty clinical cases were retrospectively used to automatically determine the best incidences of 5,7 and 9 beams plan sets with coplanar and non-coplanar geometries. The BAO and the FMO problems were addressed together by using a multicriterial IMRT optimization framework to guide the process. Algorithm $i$ is based on a combinatorial iterative discrete search approach and is embedded in the multicriterial optimization framework. Algorithm $B$ is based on a continuous space search using a pattern search method. In the final optimization phase 240 plans with 27 associated structures were generated for each algorithm. Starting from the equidistant solution, BAO plans were considered, covering an expressive universe of 3640 beam incidences, 520 plans and 14040 dosimetric structures statistics available to be analysed. The analysis of this large amount of data was done from two perspectives: the characterization of the beam angle distribution over the space search and the assessment of the quality of the dose distribution of the generated plans. To our knowledge, this is the first work that compares these two types of class methods for head and neck cancer taking into consideration all the clinical structures using subjacent clinical criteria. Furthermore, the graphical options ad-hoc constructed for this purpose - the circular diagrams for the coplanar case and the 2D-map for the non-coplanar one - are unique, enabling a global analysis that otherwise would be difficult to be performed.

The relative frequency patterns of the beam angle distribution for coplanar and non-coplanar beams geometries seemed to be conditioned by the optimization strategy followed by each algorithm. In algorithm $i$, beams with optimal orientation were iteratively added into the plan after being combined with the beams already selected in a discretized space search. For plans using coplanar beams, this cumulative beam adding methodology generated a non-uniform angle distribution pattern where it is possible to clearly identify favourite irradiation directions and regions of low preference. For non-coplanar beam plans this asymmetric beam distribution pattern with well-defined preferred incidences blurred into an almost uniform beam distribution pattern. This pattern change is a natural consequence of the selection of beam incidences over almost all the available space search. In algorithm $B$, the search for the best ensemble is initially done by considering a fixed number of incidences defined from the best equidistant coplanar angle set solution. This preliminary optimization is followed by the application of the pattern search method considering a continuous space search. Although the equidistant beam ensemble seems to be the most reasonable BAO starting point for this approach, the beam angle distribution maps presented patterns that may be strongly influenced biased by the initial solution. For coplanar geometries an almost uniform pattern, with low relative frequency values was patent in the circular diagram of frequencies. For the noncoplanar situation, the results follow the starting point option, being the non-coplanarity confined to modest deviations from zero couch position $\left( \pm 20^{\circ}\right)$. Comparing the mean incidences and the 
associated standard deviations obtained by the two optimization algorithms, once again the optimization strategy of each of the algorithms is patent, leading algorithm $i$ to more distributed incidence solutions and algorithm $B$ proposing solutions closer to the initial equidistant case.

The quality assessment and comparison of the plans generated with BAO was performed using three types of approaches: a global plan analysis, a group plan analysis and an individual analysis of selected patients. This methodology was accomplished by the determination of SPIDERplan scores and an appropriate statistical analysis and conferred to the process the possibility to evaluate the dosimetric quality of the BAO with different levels of specificity. Increasing the number of beams brought improvements to the plan dose distribution. Nevertheless, for most of the cases only when 9 beam plans were compared with 5 beam plans significant statistical score differences were found. Concerning the comparison of the two BAO methods, algorithm $B$ showed a more consistent behaviour and presented, by a moderate score difference, a better performance than algorithm $i$. For the studied NPC, on average, non-coplanarity brought no improvements to plan quality. Just for algorithm B a non-statistically significant better score was obtained when compared with the corresponding coplanar solution. These results confirmed some empirical impressions shared by many planners. In face of highly complex planning cases, beyond the manual tuning of the objectives and the associated weights, planners usually try to play with the initial beam angle incidences or to increase the number of beams in order to improve the plans. Nevertheless, BAO for the studied pathology seemed to bring only marginal improvements to the plan quality. A first explanation may be related with the anatomy of the NPC cases, where the PTVs with large extensions (up to $25 \mathrm{~cm}$ of height), the high number of critical structures along the field of irradiation and minimum exposure requirement for the remaining normal tissues may limit the optimization of the beam incidences. Another justification can be found in the use of the same wish-list for all patients and for both BAO approaches. The improvement that can be obtained by BAO is intrinsically linked to the FMO approach. Since the resources and time needed to find an optimal beam set are normally costly, if manual tuning is needed then the clinical utility of BAO has to be seriously taken into account. If BAO can be done in an automated way, then it will be surely represent an added-value, since it can bring interesting improvements for some patients. More expressive score differences between the treatment planning sets could be achieved if the SPIDERplan score could be embedded in the BAO process as suggested by Rocha et al. [26]. As SPIDERplan methodology incorporates the radiation oncologist preferences, it could confer to the BAO process some proximity to the clinical aims and thus improve the overall plan quality.

The results of algorithm $i$ deserve also to be discussed. The overall weaker performance of this algorithm, when compared with algorithm $B$, is related with the results of the non-coplanar optimization in the Optics and in the Bone groups, since for the remaining groups these sets presented the best SPIDERplan scores. For the Bone group, although the non-coplanar optimization of algorithm $i$ presented a better performance than the coplanar set of algorithm $i$, it was inferior to the coplanar optimization of algorithm $B$. For the Optics group, the results of the non-coplanar sets of algorithm $i$ were by far the worst when compared with the remaining sets. Due to the anatomic localisation of the structures of these two groups and also to the optimization methodology subjacent to algorithm $i$, it was not expected that the non-coplanar optimization presented such results that were on average bellow the score tolerance but were worse than the remaining sets.

\section{Conclusions}

In this work the beam angle optimization IMRT was addressed using forty head-and-neck clinical cases. Two algorithms, based on a combinatorial iterative (algorithm $I$ ) and on a continuous exploration of the space search (algorithm $B$ ) approaches, were assessed and compared for coplanar and non-coplanar beam geometries. A graphical method for plan quality assessment and comparison, named SPIDERplan, was used. The two algorithms were assessed through the analysis of the beam angle distribution and of the plan quality. The great amount of generated data was 
managed through creative graphical plots that enabled efficient global analysis and comparisons. Algorithm $i$ for coplanar optimization presented a less uniform angle distribution pattern whereas for non-coplanar optimization the beam distribution pattern was almost uniform. For algorithm $B$, both beam angles geometries options, presented a strong influence of the starting equidistant solution. Concerning BAO algorithms assessment and comparison, slightly better score performance was achieved by algorithm $B$, when compared to algorithm $i$. For algorithm $B$, coplanar and non-coplanar beam angle geometries were statistically equivalent, while for algorithm $i$, non-coplanar solutions were statistically worse then the correspondent coplanar. Nevertheless, these average results can be reversed for specific patient cases.

\section{Acknowledgments}

The authors would like to express their gratitude to Sebastiaan Breedveld and Ben Heijmen for making available the multicriterial optimization of IMRT plans system Erasmus-iCycle and their valuable support and coaching along the work. The authors would like also to thank to Andreia Hall for the technical support in the statistical analysis.

This work was supported by project grant POCI-01-0145-FEDER-028030 and by the Fundação para a Ciência e a Tecnologia (FCT) under project grant UID/Multi/00308/2019.

No potential conflict of interest nor any financial disclosures must be declared.

\section{References}

[1] ICRU. International Commission on Radiation Units and Measurements. Prescribing, Recording, and Reporting Photon-Beam Intensity-Modulated Radiation Therapy (IMRT). ICRU Report 83, J ICRU 2010:10(1):1-106. https://doi.org/10.1093/jicru/10.1.Report83

[2] Zhang Y, Merritt M. Dose-volume-based IMRT fluence optimization: a fast least-squares approach with differentiability. Linear Algebr Appl 2008;428;1365-87. https://doi.org/10.1016/j.laa.2007.09.037

[3] Spirou SV, Chui CS. A gradient inverse planning algorithm with dose-volume constraints. Med Phys 1998;25(3):321-33. https://doi.org/10.1118/1.598202

[4] Webb S. Optimization of conformal radiotherapy dose distributions by simulated annealing. Phys Med Biol 1989;34(10):1349-70. https://doi.org/10.1088/0031-9155/34/10/002

[5] Thieke C, Küufer KH, Monz M, Scherrer A, Alonso F, Oelfke $U$ et al. A new concept for interactive radiotherapy planning with multicriteria optimization: First clinical evaluation. Radiother and Oncol 2007;85(2):292-98. https://doi.org/10.1016/j.radonc.2007.06.020

[6] Miettinen K. Nonlinear multiobjective optimization. New York: Springer US; 1998. https://doi.org/10.1007/9781-4615-5563-6

[7] Branke J, Deb K, Miettinen K, Slowiński R. Multiobjective Optimization. Interactive and Evolutionary Approaches. 1st ed. New York: Springer-Verlag Berlin Heidelberg; 2008. https://doi.org/10.1007/978-3-54088908-3

[8] Das SK, Marks LB. Selection of coplanar or noncoplanar beams using three-dimensional optimization based on maximum beam separation and minimized nontarget irradiation. Int J Radiat Oncol Biol Phys 1997;38(3):64355. https://doi.org/10.1016/S0360-3016(97)89489-8

[9] Rocha H, Dias J, Ventura T, Ferreira B, Lopes MC. A derivative-free multistart framework for an automated noncoplanar beam angle optimization in IMRT. Med Phys 2016;43(10):5514-26. https://doi.org/10.1118/1.4962477.

[10] Bortfeld T, Schlegel W. Optimization of beam orientations in radiation therapy: some theoretical considerations. Phys Med Biol 1993;38(2):291-304. https://doi.org/10.1088/0031-9155/38/2/006

[11] Ehrgott M, Holder A, Reese J. Beam selection in radiotherapy design. Linear Algebra Appl 2008;428(5):1272312. https://doi.org/10.1016/j.laa.2007.05.039

[12] Craft D. Local beam angle optimization with linear programming and gradient search. Phys Med Biol 2007;52(7):127-35. https://doi.org/10.1088/0031-9155/52/7/N02

[13] Stein J, Mohan R, Wang XH, Bortfeld T, Wu Q, Preiser K et al. Number and orientation of beams in intensitymodulated radiation treatments. Med Phys 1997;24(2):149-60. https://doi.org/10.1118/1.597923

[14] Pugachev A, Lei X. Pseudo beam's-eye-view as applied to beam orientation selection in intensity-modulated radiation therapy. Int J Radiat Oncol Biol Phys 2001;51(5):1361-70. https://doi.org/10.1016/S03603016(01)01736-9

[15] Dias J, Rocha H, Ferreira BC, Lopes MC. Simulated annealing applied to IMRT beam angle optimization: A computational study. Physica Med 2015;31(7):747-56. https://doi.org/10.1016/j.ejmp.2015.03.005

[16] Dias J, Rocha H, Ferreira BC, Lopes MC. A genetic algorithm with neural network fitness function evaluation for IMRT beam angle optimization. Cent Eur J Oper Res 2014;22(3):431-55. https://doi.org/10.1007/s10100013-0289-4 
[17] Aleman DM, Kumar A, Ahuja RK, Romeijn HE, Dempsey JF. Neighborhood search approaches to beam orientation optimization in intensity modulated radiation therapy treatment planning. J Global Optim 2008;42(4):587-607. https://doi.org/10.1007/s10898-008-9286-x

[18] Lim GJ, Cao W. A two-phase method for selecting IMRT treatment beam angles: Branch-and-Prune and local neighborhood search. Eur J Oper Res 2012;217(3):609-18. v https://doi.org/10.1016/j.ejor.2011.09.038

[19] Bertsimas D, Cacchiani V, Craft D, Nohadani O. A hybrid approach to beam angle optimization in intensitymodulated radiation therapy. Comput Oper Res 2013;40(9):2187-97. https://doi.org/10.1016/j.cor.2012.06.009

[20] Sultan ASA. Optimization of beam orientations in intensity modulated radiation therapy planning PhD Thesis. Department of Mathematics, Technical University of Kaiserslautern, Germany; 2006.

[21] Lim GJ, Choi J, Mohan R. Iterative solution methods for beam angle and fluence map optimization in intensity modulated radiation therapy planning. OR Spectrum 2008;30(2):289-309. https://doi.org/10.1007/s00291-0070096-1

[22] Breedveld S, Storchi $P$, Voet $P$, Heijmen B. iCycle: integrated, multi-criterial beam angle and profile optimization for generation of coplanar and non-coplanar IMRT plans. Med Phys 2012;39(2):951-63. https://doi.org/10.1118/1.3676689

[23] Bangert M, Unkelbach J. Accelerated iterative beam angle selection in IMRT. Med Phys 2016;43(3):1073-82. https://doi.org/10.1118/1.4940350

[24] Rocha H, Dias J, Ferreira BC, Lopes MC. Beam angle optimization for intensity-modulated radiation therapy using a guided pattern search method. Phys Med Biol 2013;58(9):2939-53. https://doi.org/10.1088/00319155/58/9/2939

[25] Rocha H, Dias JM, Ventura T, Ferreira B, Lopes M. A derivative-free multistart framework for an automated noncoplanar beam angle optimization in IMRT. Med Phys 2016;43(10):5514-26. https://doi.org/10.1118/1.4962477

[26] Rocha H, Dias JM, Ventura T, Ferreira B, Lopes M. Beam angle optimization in IMRT: are we really optimizing what matters? Intl. Trans in Op Res 2019;26(3):908-28. https://doi.org/10.1111/itor.12587

[27] Breedveld S, Storchi P, Keijzer M, Heemink A, Heijmen B. A novel approach to multi-criteria inverse planning for IMRT. Phys Med Biol 2007;52(20):6339-53. https://doi.org/10.1088/0031-9155/52/20/016

[28] Ventura T, Lopes MC, Ferreira BC, Khouri L. SPIDERplan: A tool to support decision-making in radiation therapy treatment plan assessment. Reports Pract Oncol Radiother 2016;21(6):508-16. https://doi.org/10.1016/j.rpor.2016.07.002

[29] Breedveld S, Storchi $P$, Heijmen B. The equivalence of multi-criteria methods for radiotherapy plan optimization. Phys Med Biol 2009;54(23):7199-209. https://doi.org/10.1088/0031-9155/54/23/011

[30] Custódio AL, Rocha H, Vicente LN. Incorporating minimum Frobenius norm models in direct search. Comput Optim Appl 2010;46:265-78. https://doi.org/10.1007/s10589-009-9283-0 